\title{
Reducing Radar Cross Section by Investigation Electromagnetic Materials
}

\author{
Somayeh Komeylian $^{1}$, Saeed Komeylian ${ }^{2}$, Farokh Hojjat Kashani ${ }^{3}$ \\ ${ }^{1}$ School of Electrical Engineering, Islamic Azad University south Tehran Branch, Tehran, Iran \\ ${ }^{2}$ Department of Electrical Engineering, Sharif University of Technology, Tehran, Iran \\ ${ }^{3}$ School of Electrical Engineering, University of Iran Science \& Technology, Tehran, Iran \\ E-mail: somayeh@komeylian.com, saeed@komeylian.com, kashani@iust.ac.ir
}

\begin{abstract}
Decreasing the Radar Cross Section (RCS) is investigation in electromagnetic materials, i.e. double-positive (DPS), double-negative (DNG), epsilon-negative (ENG), and munegative materials lead to a great motive in manufacturing structures with unusual electromagnetic characteristics. The valid conditions for achieving the transparency and gaining resonance for an electrically small cylinder are established, in this correspondence. The effect of incidence direction on RCS inclusive of transparency and resonance conditions is also explored, through computer simulation for an electrically small cylinder.
\end{abstract}

\section{Introduction}

Recently, reducing the Radar Cross Section (RCS) of various structures for achieving transparency, and obtaining resonant structures have received meticulous attention. For instance, in [1-4], the transparency conditions for typical objects of cylindrical and spherical shapes were established. The resonance conditions of such objects were also considered in $[8,9]$. Now, we aim to derive and elucidate these unusual particularizes of this group of electromagnetic materials, Fig. 1, for electrically small cylindrical object $[6$, 7].

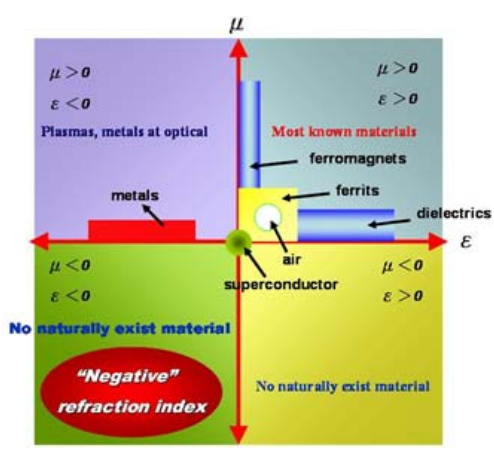

Figure 1: Materials classification.

Invisibility means that an object doesn't obstruct what is behind it, and its scattering cross section is significantly low, at least in a narrow frequency band. Therefore, by passing the light through these structures, under transparency phenomena scattering, distortion, or observation are dramatically decreased. In this paper, we are interested in extracting the transparency conditions for an electrically small cylinder for both polarizations, i.e. TE, and TM.

An electrically small object generally shows a weak scattering for any incident (plane) wave. A peak in the scattering cross section of such an object which depends on its polarizations means that a resonance. We can use Metamaterials for gaining an unusual scattering, i.e. huge scattering resonance. Another task of this paper is to derive resonance conditions for this electrically small cylinder, and for both polarizations.

In addition to what was stated above, RCS is related to not only transparency, and resonance conditions, but also the direction incidence. Therefore, we next illustrate the effect of direction incidence on RCS under transparency, and resonance conditions.

Numerical results are provided in the form of normalized bistatic echo widths.

\section{Theoretical background for normalized bistatical echo width}

Consider a plan wave obliquely incidence with TM polarization on an electrically small cylinder with the radius 'a', and infinite length as indicated in Fig. 2.

By referring to Fig. 2 the incident electric field can be written as follows.

$$
\begin{gathered}
E^{i}=\left(\hat{a}_{x} E_{0} \cos \theta_{0} \cos \varphi_{0}+\hat{a}_{y} E_{0} \cos \theta_{0} \sin \varphi_{0}+\hat{a}_{z} E_{0} \sin \theta_{0}\right) . \\
e^{-j k_{0} x \sin \theta_{0} \cos \varphi_{0}} e^{-j k_{0} y \sin \theta_{0} \cos \varphi_{0}} e^{-j k_{0} z \cos \theta_{0}},
\end{gathered}
$$

By substituting $x=\rho \cos \varphi$, and $y=\rho \sin \varphi$, the $Z$ component of the electric field will be obtained by following expansions.

$$
\begin{gathered}
E_{z}^{i}=E_{0} \sin \theta_{0} e^{-j k_{0} \rho \sin \theta_{0}\left(\cos \varphi_{0} \cos \varphi+\sin \varphi \sin \varphi_{0}\right)}= \\
E_{0} \sin \theta_{0} e^{-j k_{0} \rho \sin \theta_{0} \cos \left(\varphi-\varphi_{0}\right)} e^{j k_{0} z \cos \theta_{0}},
\end{gathered}
$$

The factor $e^{-j k_{0} \rho \cos \left(\varphi-\varphi_{0}\right)}$ can be rewritten by an infinite sum of cylindrical wave functions as follows. 
$e^{-j k_{0} \rho \cos \left(\varphi-\varphi_{0}\right)}=\sum_{n=-\infty}^{+\infty} a_{n} J_{n}\left(k_{0} \rho\right) e^{j n \varphi_{0}}$,

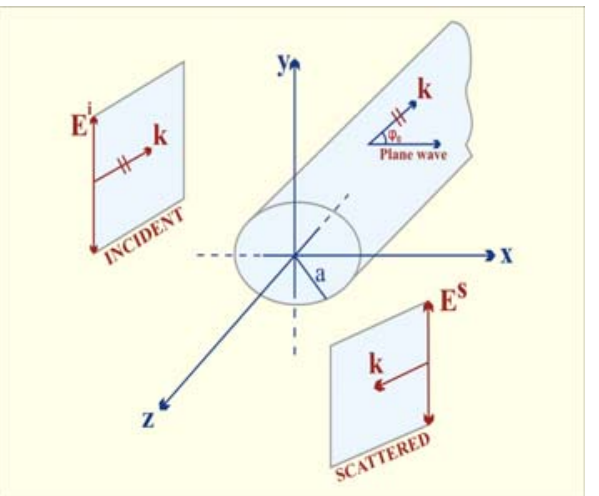

Figure 2: Uniform plane wave obliquely incident on a metamaterials cylinder: $\mathrm{TM}^{\mathrm{z}}$ polarization.

By applying equation (3) into the equation (2), the following expression will be gained.

$$
E_{z}^{i}=E_{0} \sin \theta_{0} e^{-j k_{0} z \sin \theta_{0}} \sum_{n=-\infty}^{+\infty} j^{-n} J\left(k_{0} \rho \sin \theta_{0}\right) e^{\left(\varphi-\varphi_{0}\right)},
$$

Because of the cylinder has an infinite length, the $\mathrm{Z}$ component of the fields are periodic and vary according to the factor $e^{-j k_{0} Z \cos \theta_{0}}$

The $\mathrm{Z}$ components of the scattered and transmitted electric fields would be derived by the similar procedure in incident electric field as follow.

$$
\begin{aligned}
& E_{z}^{s}=E_{0} \sin \theta_{0} e^{-j k_{0} \cos \theta_{0}} \sum_{n=-\infty}^{+\infty} j^{-n} C_{n} H_{n}^{(2)}\left(k_{0} \rho \sin \theta_{0}\right) e^{j n\left(\varphi-\varphi_{0}\right)} \\
& E_{z}^{t}=E_{0} \sin \theta_{0} e^{+j k_{0} z \cos \theta_{0}} \sum_{n=-\infty}^{+\infty} j^{-n} a_{n} J_{n}\left(k \rho \sin \theta_{1}\right) e^{j n\left(\varphi-\varphi_{0}\right)}
\end{aligned}
$$

By satisfying the Maxwell's equations, the longitudinal magnetic field components for the scattered and transmitted waves will be expressed by the following equations.

$$
\begin{aligned}
& H_{z}^{i}=0, \\
& H_{z}^{s}=E_{0} \sin \theta_{0} e^{+j k_{0} z \cos \theta_{0}} \sum_{n=-\infty}^{+\infty} j^{-n} \tilde{c}_{n} H_{n}^{(2)}\left(k_{0} \rho \sin \theta_{0}\right) e^{j n\left(\varphi-\varphi_{0}\right)}, \\
& H_{z}^{t}=E_{0} \sin \theta_{0} e^{j k_{0} z \cos \theta_{0}} \sum_{n=-\infty}^{+\infty} j^{-n} \tilde{a}_{n} J_{n}\left(k \rho \sin \theta_{1}\right) e^{j n\left(\varphi-\varphi_{0}\right)},
\end{aligned}
$$

Because of phase matching, the propagation constant in the longitudinal direction, $\mathrm{k}_{\mathrm{z}}$, should be the same as free space, and Metamaterial media which has indicated in Fig. 3 , will be expressed in the following expressions.

$$
\begin{gathered}
k_{0} \cos \theta_{0}=k \cos \theta_{1}, \\
\cos \theta_{1}=\frac{k_{0}}{k} \cos \theta_{0}, \\
\sin \theta_{0}=\left\{\begin{array}{ll}
\sqrt{1-\left(\frac{k_{0}}{k}\right)^{2} \cos ^{2} \theta_{0}} & \left|\frac{k_{0}}{k} \cos \theta_{0}\right| \leq 1 \\
-j \sqrt{\left(\frac{k_{0}}{k}\right)^{2} \cos ^{2} \theta_{0}-1} & \left|\frac{k_{0}}{k} \cos \theta_{0}\right|>1
\end{array}\right\},
\end{gathered}
$$

And

$$
k_{t}=k \sin \theta_{1},
$$

Where $K_{t}$ is the transverse propagation constant in Metamaterial medium.

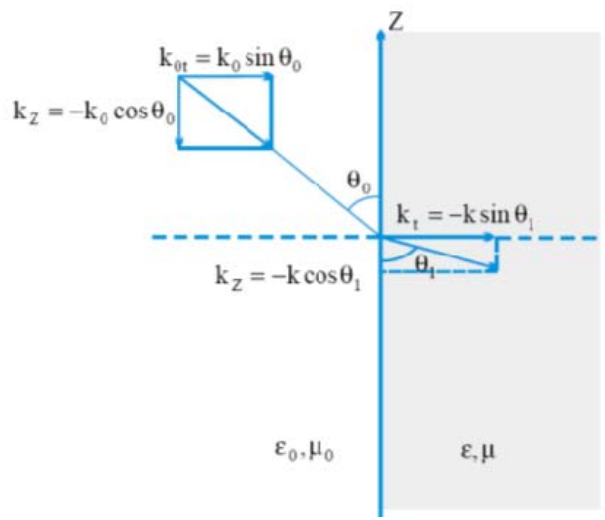

Figure 3: Longitudinal and transverse components of the incident and transmitted fields.

The unknown coefficients $a_{n}^{T M}, b_{n}^{T M}$ and $c_{n}^{T M}$ are determined from applying boundary conditions. Tangential components of the electric and magnetic fields should be continuous as indicated that in the following expressions.

$$
\begin{aligned}
& E_{z}^{i}(\rho=a)+E_{z}^{s}(\rho=a)=E_{z}^{t}(\rho=a), \\
& H_{z}^{i}(\rho=a)+H_{z}^{s}(\rho=a)=H_{z}^{t}(\rho=a), \\
& E_{\varphi}^{i}(\rho=a)+E_{\varphi}^{s}(\rho=a)=E_{\varphi}^{t}(\rho=a), \\
& H_{\varphi}^{i}(\rho=a)+H_{\varphi}^{s}(\rho=a)=H_{\varphi}^{t}(\rho=a),
\end{aligned}
$$

The $\varphi$ components of the incident, scattered, and transmitted electrical and magnetic fields will be derived from their Z components by applying Maxwell's Equations [5].

The large argument approximations of Hankel's and Bessel's functions will be used for far field zone for both scattered and transmitted electrical fields so that normalized bistatic echo width will be realized as follows.

$$
\begin{aligned}
& \frac{\sigma^{T M}}{\lambda_{0}}=\lim _{\rho \rightarrow \infty} \frac{\left[2 \pi \rho \frac{\left|E_{z}^{s}\right|^{2}}{\left|E_{z}^{j}\right|^{2}}\right]}{\lambda_{0}} \\
& =\frac{2}{\pi \sin \theta_{0}}\left\{\left|\sum_{n=-\infty}^{+\infty} c_{n}^{T M} e^{j n\left(\varphi-\varphi_{0}\right)}\right|^{2}+\frac{1}{\eta_{0}^{2}}\left|\sum_{n=-\infty}^{+\infty} \tilde{c}_{n}^{T M} e^{j n\left(\varphi-\varphi_{0}\right)}\right|^{2}\right\},
\end{aligned}
$$

Likewise, the unknown coefficient, $\tilde{C}_{n}$ are gained by applying boundary conditions. Under consideration at normal condition, i.e. $\varphi=0^{0}$ or $\theta_{0}=90^{\circ}$, the equation (14) will be changed into the following equation.

$$
\begin{aligned}
& \frac{\sigma^{T M}}{\lambda_{0}}=\frac{2}{\pi}\left|\sum_{n=-\infty}^{+\infty} c_{n}^{T M} e^{j n\left(\varphi-\varphi_{0}\right)}\right|^{2}, \\
& \text { Where } c_{n}^{T M}, \text { is. }
\end{aligned}
$$


$c_{n}^{T M}=\frac{\zeta J_{n}(k a) J_{n}^{\prime}\left(k_{0} a\right)-J_{n}^{\prime}(k a) J_{n}\left(k_{0} a\right)}{J_{n}^{\prime}(k a) H_{n}^{(2)}\left(k_{0} a\right)-\zeta J(k a) H_{n}^{(2)}\left(k_{0} a\right)}$,

Where $\zeta=\sqrt{\frac{\mu_{c} \varepsilon_{0}}{\mu_{0} \varepsilon_{c}}}$.

The procedure for $T E^{Z}$ polarization is the same as the $T M^{z}$ polarization, but electrical field will be replaced with magnetic field and then their corresponding boundary conditions will be applied, Fig. 4.

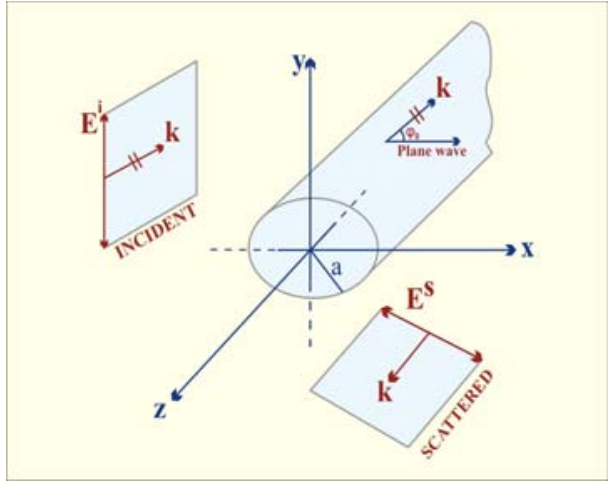

Figure 4: Uniform plane wave obliquely incident on a Metamaterials cylinder: $T E^{z}$ polarization.

Thus the formulations of RCS remain the same as TM polarization except for $c_{n}^{T E}$ which is found by the following form.

$c_{n}^{T E}=\frac{J_{n}(k a) J_{n}^{\prime}\left(k_{0} a\right)-\zeta J_{n}^{\prime}(k a) J\left(k_{0} a\right)}{\zeta J_{n}^{\prime}(k a) H_{n}^{(2)}(k a)-J_{n}(k a) H_{n}^{(2)}\left(k_{0} a\right)}$,

\section{Transparency for electrically small cylinder}

The transparency conditions for an electrically small cylinder will be derived for both polarizations by setting the numerators of the scattering coefficients given in the equations (16), and (17) to zero. In the sub-wavelength limit assuming $|k| a \square 1$, and $k_{0} a \square 1$ and applying the small argument forms of Bessel and Hankel functions will lead to the following transparency conditions as follows.

For TM polarization:

$c_{n}^{T M}=0 \rightarrow \zeta J_{n}(k a) J_{n}^{\prime}\left(k_{0} a\right)-J_{n}^{\prime}(k a) J_{n}\left(k_{0} a\right)=0$,

Under consideration of $|k| a \square 1, \quad$ and $\quad k_{0} a \square 1$ conditions we could use the approximations of Bessel functions as follows.

$$
\begin{aligned}
\zeta \times \frac{1}{n !}\left(\frac{k a}{2}\right)^{n} \times \frac{1}{2} \frac{1}{(n-1) !} \frac{\left(k_{0} a\right)^{n-1}}{2}= \\
\frac{1}{2} \frac{1}{(n-1) !}\left(\frac{k a}{2}\right)^{n-1} \times \frac{1}{n !} \frac{\left(k_{0} a\right)^{n}}{2},
\end{aligned}
$$

By simplifying the last equation, the below equation would be found.

$$
\zeta k=k_{0},
$$

By substituting the values of $\mathrm{k}$ and $\mathrm{k}_{0}$ we can derivate the given form.
For TE polarization:

$$
\mu_{c}=\mu_{0},
$$

$c_{n}^{T E}=0 \rightarrow J_{n}(k a) J_{n}^{\prime}\left(k_{0} a\right)-\zeta J_{n}^{\prime}(k a) J\left(k_{0} a\right)=0$,

By applying the approximation of Bessel's functions as follows.

$$
\begin{aligned}
& \frac{1}{n !}\left(\frac{k a}{2}\right)^{n} \times \frac{1}{2} \frac{1}{(n-1) !}\left(\frac{k_{0} a}{2}\right)^{n-1}= \\
& \zeta \times \frac{1}{2} \frac{1}{(n-1) !}\left(\frac{k a}{2}\right) \times \frac{1}{n !}\left(\frac{k_{0} a}{2}\right)^{n},
\end{aligned}
$$

By simplifying the above equation, we have the following form.

$$
\zeta k_{0}=k
$$

By substituting the values of $\mathrm{k}$, and $\mathrm{k}_{0}$, we can derivate the bellow form.

$$
\varepsilon_{c}=\varepsilon_{0},
$$

Therefore, for TM polarization, in order to get results using of DPS or MNG materials could be realized. Moreover, for TE polarization applying of DPS or ENG material would be found. Numerical results to verify the analytical derivations are fulfilled by Matlab simulation in Fig. 5.

Transparency condition doesn't result from resonation so that it will not very sensitive to ohmic losses, but for ENG material, there is high sensitivity to ohmic losses near the resonance.

(a) $\varepsilon_{r}=1+0.01 j, \theta_{0}=90^{\circ}$

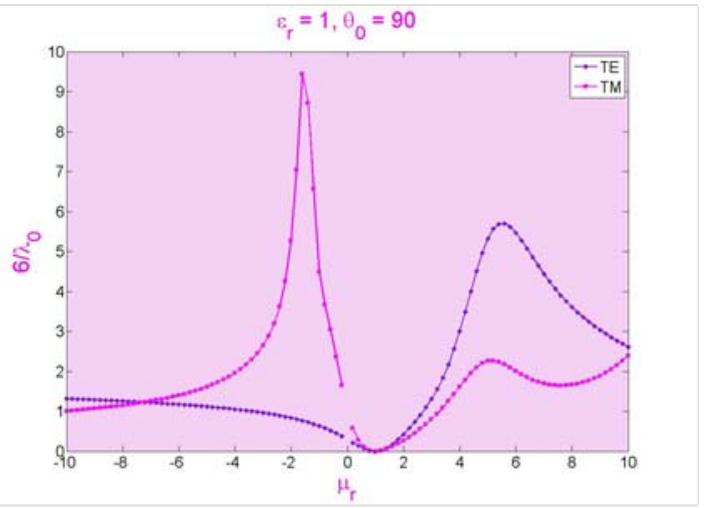

(b) $\mu_{r}=1+0.01 j, \theta_{0}=90^{\circ}$

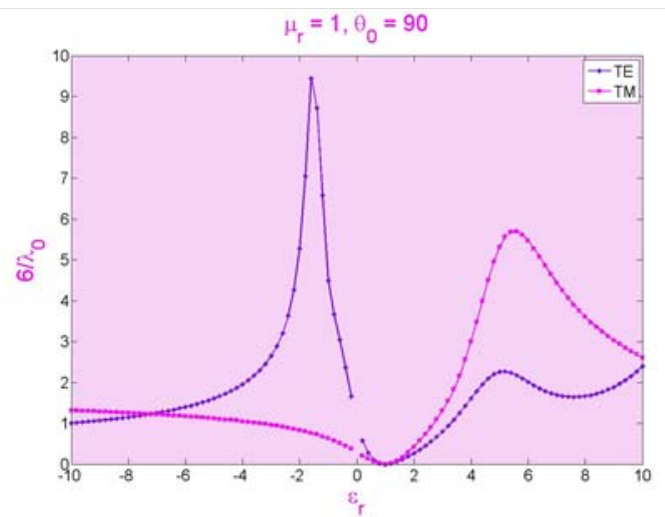

Figure 5: Normalized monostatic echo width of cylinder $(\mathrm{a}=5 \mathrm{~mm}, \mathrm{f}=1 \mathrm{GH})$ for transparency conditions or RCS minimization. 


\section{Resonant electrically small cylinder}

The resonance conditions for an electrically small cylindrical scattered is derived for both polarizations by setting the denominator of the scattering coefficients given in equations (16), and (17) to zero. This results in the maximum RCS, and yields the following resonance conditions.

For TM polarization:

$$
c_{n}^{T M} \rightarrow \infty \rightarrow J_{n}^{\prime}(k a) H_{n}^{(2)}\left(k_{0} a\right)-\zeta J_{n}(k a) H_{n}^{\prime(2)}\left(k_{0} a\right)=0,
$$

According to the supposition of $|\mathrm{k}|<<1$, and $\mathrm{k}_{0} \mathrm{a}<<1$, and applying the approximation of Bessel's functions, the following equation would be rewritten.

$$
\begin{aligned}
& \frac{1}{2} \times \frac{1}{(n-1) !}\left(\frac{k a}{2}\right)^{n-1} \times j\left(\frac{2}{k_{0} a}\right)^{n} \frac{(n-1) !}{\pi}= \\
& \zeta \times \frac{1}{n !}\left(\frac{k a}{2}\right)^{n}(-j) \frac{2^{n}}{\pi} \frac{n !}{\left(k_{0} a\right)^{n+1}},
\end{aligned}
$$

By the simplifying the last equation, the following expression could be realized.

$$
k_{0}=-\zeta k \text {, }
$$

By substituting the values of the $\mathrm{k}$, and $\mathrm{k}_{0}$ the bellow result could be found.

$$
\mu_{c}=-\mu_{0},
$$

For TE polarization:

$c_{n}^{T E} \rightarrow \infty \rightarrow \zeta J_{n}^{\prime}(k a) H_{n}^{(2)}\left(k_{0} a\right)-J_{n}(k a) H_{n}^{\prime(2)}\left(k_{0} a\right)=0$,

Based on the same assumption, we could apply the approximation of Hankel functions as follows.

$$
\begin{array}{r}
\zeta \times \frac{1}{2} \frac{1}{(n-1) !}\left(\frac{k a}{2}\right)^{n-1} \times j\left(\frac{2}{k_{0} a}\right)^{n} \frac{(n-1) !}{\pi}= \\
\frac{1}{n !}\left(\frac{k a}{n}\right)^{n} \times(-j) \frac{2^{n}}{\pi} \frac{n !}{\left(k_{0} a\right)^{n+1}},
\end{array}
$$

By simplifying the above relationships we can derive the expression.

$$
\zeta k_{0}=-k,
$$

By substituting the values of $k$, and $\mathrm{k}_{0}$ we can extract the following equation.

$$
\varepsilon_{c}=-\varepsilon_{0}
$$

Therefore, for TM polarization, for the best result employing the MNG, and DNG materials are realized. Moreover, for TE polarization applying the ENG and DNG materials for the best results is understood. Fig. 6 .

The major restrictions on using metallic structures are their conductivity loss, and difficulties of fabrications. Furthermore, most of the known such materials have inherent anisotropic peculiarities, and narrow band width resonance.

The merits of employing mere dielectric Metamaterials in comparison with metallic materials are feasibility for fabrication, having no metallic loss, and designing by means of isotropic Metamaterials. In order to get results, and high efficiency, the Metamaterials have received a majority of candidates. (a) $\varepsilon_{r}=-1+0.01 j, \theta_{0}=90^{\circ}$

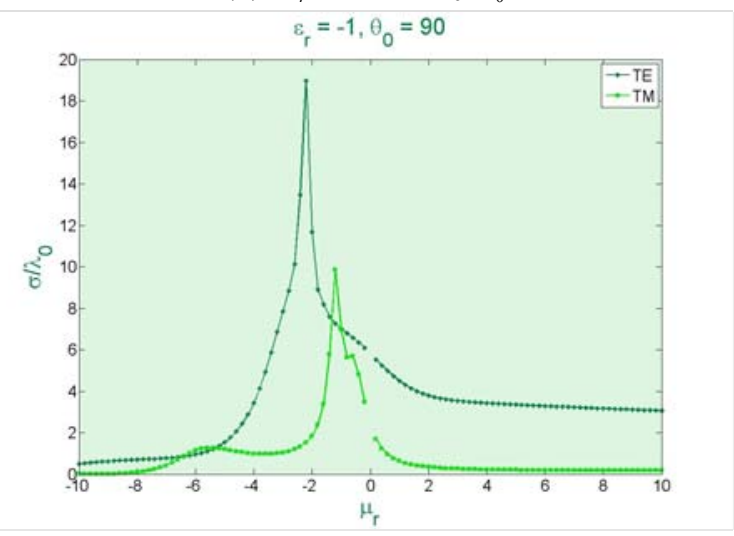

(b) $\mu_{r}=-1+0.01 j, \theta_{0}=90^{\circ}$

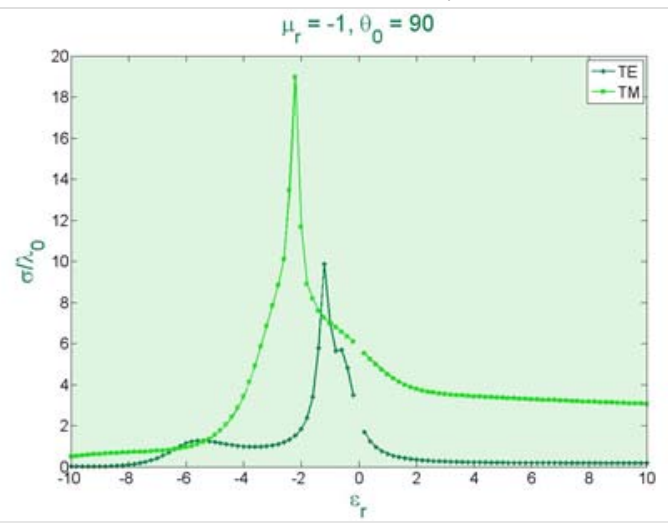

Figure 6: Normalized monostatic echo width of Metamaterial cylinder $(a=5 \mathrm{~mm}, \mathrm{f}=1 \mathrm{GHz})$ for resonance condition or RCS maximization.

\section{The bistatic normalized echo widths}

Bistatic normalized echo widths inclusive of transparency, and resonance conditions for both TE and TM polarizations are performed by Matlab simulation. Under resonance condition, Fig. 7-b, the minimum RCS has happened in both incident, and backscattering directions, and almost at $\varphi=90^{\circ}$.

Under transparency condition, Fig. 7-a, the maximum RCS has occurred dramatically in the incident direction, i.e. $\varphi=0^{0}$, after that in the backscattering direction, i.e. $\varphi=180^{\circ}$.

There are some considerations for Bistatic normalized echo widths as follows.

- On the increase the backscattering will be increased when the wavelength of the incident wave corresponds to the inner wavelength of the scatters, e.g. the crystal lattice space of crystal, or the wavelength of subwavelength resonant metallic element, etc.

- On the other hand, the practical results show that the amount of backscattering is related to wind spaced, the wave length of the surface interaction, and etc. rather than the relationship of the theoretical estimates. 
a)

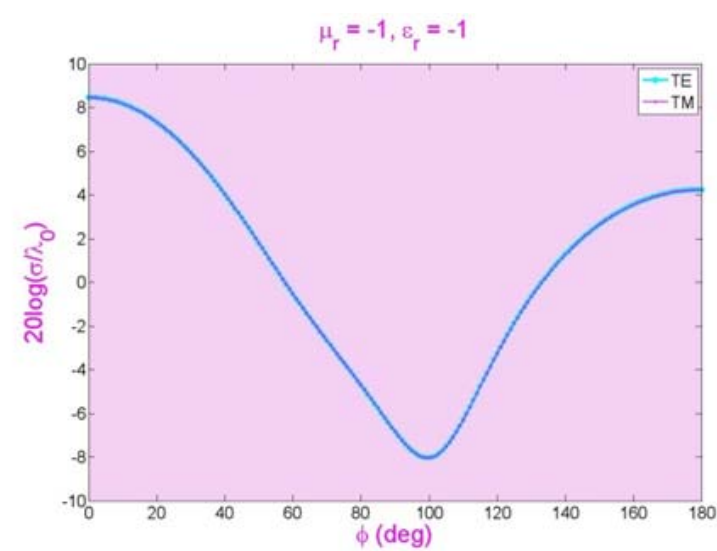

b)

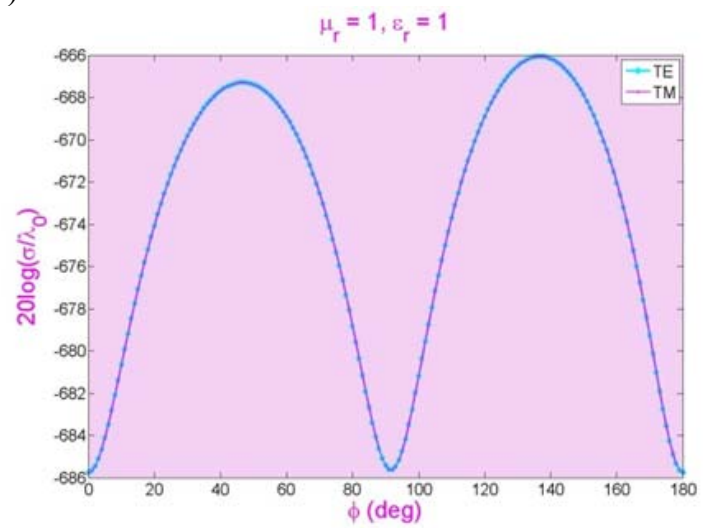

Figure 7: Normalized bistatic echo widths for (a) DPS materials (b) Metamaterials.

\section{Some considerations in design}

In this work, we are persevering in our effects to ascertain the behavior of two phenomena, i.e. resonance, and transparency conditions.

To design a material with a desired manner it is necessary to meticulous attention to the dielectric, and magnetic behavior of Fig. 5, and according to the desired electric and magnetic behavior, creat appropriate electric, and magnetic dipole moment in small size. It is require to trade off the resonant condition with transparency condition during the design. Basically, the electric and magnetic dipole moments should be considered as the alphabet of making these materials. Most of these materials are constructed of sub-wavelength resonant metallic elements which have some drawbacks as follow:

- Having conductive loss

- Mechanical, and technical difficulties in fabricating

- Resonating at a very narrow band width

- Having an isotropic electromagnetic response at most of them.

There merits of employing mere dielectric materials in comparison with resonant metallic elements are as follows:

- Having increased efficiency because of no having the metallic loss.
- Preferable to fabricating these materials from RF to optical frequency ranges.

- Suppressing the radiation loss which each of resonators procedures individually by the periodic structure.

The value of RCS is not only depending on the backscattering direction, but also on the incidence direction.Fig.7-b.

\section{Conclusions}

The goal of this paper, as we have elucidated, is to provide a comprehensive investigation of the given materials for an electrically small cylinder to achieve the best invisibility, and resonance conditions. For the given object, $\mu_{\mathrm{c}}=\mu_{0}$ has derived as the transparency condition for TM polarization which is yielded to utilized the MNG, and DPS materials.

Furthermore, $\varepsilon_{\mathrm{c}}=\varepsilon_{0}$ has calculated for the best result of the resonance condition of TE polarization which is achieved to use the ENG, and DPS materials. On the other hand, $\mu_{c}=\mu_{0}$ has extracted as the result of huge scattering for $\mathrm{TM}$ polarization which applying of the $\mathrm{MNG}$, and $\mathrm{DNG}$ materials is concluded. In addition to $\varepsilon_{c}=\varepsilon_{0}$ has computed for resonance condition of TE polarization which employing of ENG, and DNG materials are inferred.

The bistatic echo widths are illustrated for both TE and TM polarizations under transparency, and resonance conditions through the computer simulation. The clear inference shows the dependency of maximum RCS on both incidence, and backscattering direction.

\section{Acknowledge}

I would like to express my sincere gratitude to Pro.Hojjat Kashani, for his expert, sincere, and valuable guidance extended to me.

I also place on record, the deepest appreciative to my mother for her unceasing encouragement, and support.

\section{References}

[1] A. Alù, and N. Engheta, Achieving transparency with plasmonic and coating, Phys. Rev. E 72, 016623 (2005).

[2] A. Alù and N. Engheta, Cloaking and transparency for collections of particles with Metamaterial and plasmonic, Optics Express, 7578, Vol. 15, No. 12, pp.7578-7590, 2007.

[3] E. Irci and Vakur B. Ertürk, Investigation of metamaterials coated conducting cylinders for achieving transparency and maximizing Radar Cross Section, Antenna and Propagation Society International Symposium, Honlulu, USA, pp. 857-860, 2008.

[4] Joshua A. Grodon and R.W. Ziolkowski, CNP optical Metamaterials, Optics Express, Vol. 16, No. 9, 2008.

[5] E. Iric, Wave propagation in Meatamaterial structures and retrieval of homogenization parameters, Master thesis, Billend University, 2007.

[6] A. Alù, N. Engheta, A. Erentok, R.W. Ziolkowski, Single-negative, Double-negative, and Low-index Metamaterials and their electromagnetic applications, 
IEEE Transactions on Antenna and Propagation Magazine, Vol. 49, pp. 23-36, 2007.

[7] A. Ahmadi, Metamaterials demonstrating focusing and radiation characteristics applications, $\mathrm{PhD}$. Thesis, Northeastern University, USA, 2010.

[8] A. Alù, N. Engheta, Resonances in sub-wavelength cylindrical structures made of pairs of double-negative and double-positive or epsilon-negative and munegative coaxial shells, Int. Conf. on Electromagnetics and Advanced Applications, Turin, Italy, pp. 435-438, 2008.

[9] A. Alù, N. Engheta, Sub-wavelength resonance structures containing double-negative (DNG) or singlenegative (SNG) media: Planar, cylindrical and spherical cavities, waveguides, and open scatters, Progress in Electromagnetic Research Symp., Honolulu, Waikiki, Hawaii, USA, p.12, 2003.

[10] C.A. Balanis, Advanced Engineering Electromagnetics, New York, Wiley, Ch.11, pp. 595-596, 1989.

[11]C. Li and Z. Shen, Electromagnetic scattering by a conducting cylinder coated with Metamaterials, Progress in Electromagnetics Research, Vol. 42, pp.91$105,2003$. 